\title{
Blessures et port du casque au cours d'activités non motorisées sur roues chez des patients pédiatriques
}

\author{
H. Lindsay, M.D. (1, 2); M. Brussoni, Ph. D. $(2,3,4,5,6)$
}

Cet article a fait l'objet d'une évaluation par les pairs.

Résumé

Introduction : Les patients qui se présentent aux urgences en raison de traumatismes subis au cours d'activités récréatives constituent une source d'information unique qui peut orienter de manière déterminante les interventions visant à prévenir les blessures. Nous décrivons ici l'épidémiologie des blessures associées aux activités non motorisées sur roues chez les patients pédiatriques se présentant aux urgences au Canada et faisons état du port du casque chez ces patients.

Méthodologie : Nous avons extrait les données relatives aux années 2004 à 2009 du Système canadien hospitalier d'information et de recherche en prévention des traumatismes (SCHIRPT), un programme national de surveillance des blessures dans les urgences de 15 hôpitaux.

Résultats : La majorité des 28618 enfants âgés de 1 à 16 ans qui ont été blessés au cours d'activités non motorisées sur roues l'ont été durant la pratique de la bicyclette, la deuxième cause étant la pratique de la planche à roulettes. Les enfants blessés étaient majoritairement des garçons. Les enfants blessés en trottinette étaient généralement plus jeunes, ceux blessés en planche à roulettes plus âgés. En moyenne, le nombre total de blessures a diminué de $6 \%$ au cours de la période étudiée. Les chutes étaient le mécanisme lésionnel le plus courant, et 8,3\% des patients présentaient un traumatisme crânien, blessure plus fréquente chez les cyclistes que chez les utilisateurs d'autres dispositifs sur roues. Le port du casque était plus répandu chez les cyclistes $(62,2 \%)$, les utilisateurs de planche à roulettes affichant le plus faible taux d'utilisation $(32,9 \%)$. Les patients blessés qui s'étaient présentés aux urgences dans une région où le port du casque était obligatoire étaient 2,12 fois plus susceptibles de porter un casque et 0,86 fois moins susceptibles de souffrir d'un traumatisme crânien, comparativement aux patients des régions où il n’y avait pas de réglementation sur le port du casque.

Conclusion : Ces résultats corroborent les études selon lesquelles la réglementation rendant obligatoire le port du casque pourrait être un moyen efficace de réduire les blessures chez tous les utilisateurs de dispositifs à roues non motorisés. Le petit nombre de patients qui portaient un casque et un équipement de protection (59,4\% en tout) laisse penser que cela demeure un champ d'intervention.

Mots-clés : enfant, bicyclette, patin à roues alignées, planche à roulettes, trottinette, port du casque

\section{Introduction}

Les activités non motorisées sur roues conduisent à une importante morbidité et mortalité liées aux blessures chez les enfants $^{1,2}$. Selon le Registre national des traumatismes, 27589 personnes ont été hospitalisées au Canada en raison de blessures associées à des activités sur roues entre 2004 et 2009 et, de ce nombre, 22023 étaient cyclistes ${ }^{2}$. Toutefois, ces chiffres représentent uniquement les hospitalisations et ne tiennent pas compte de l'ensemble des répercussions sur le système de santé, car bon nombre de blessures sont traitées seulement aux urgences ou en consultations externes (par exemple fractures et blessures mineures à la tête).

Plusieurs études comparant les profils de blessure associés à différentes activités sur roues ont fait ressortir des différences en fonction de l'âge ${ }^{3-5}$. Des études antérieures laissent penser que, même si les cyclistes subissent un plus grand nombre de fractures en raison de la popularité de cette activité, la proportion de fractures associées à l'utilisation des autres dispositifs non motorisés à roues est plus importante $^{6}$. Les taux de fracture chez les enfants blessés à bicyclette qui se sont présentés aux urgences canadiennes se situaient entre $26,3 \%$ et $28,9 \%^{7,8}$, alors qu'une étude norvégienne portant sur les dispositifs à roues a révélé que $69 \%$ des utilisateurs de patins à roues alignées, $48 \%$ des utilisateurs de planches à roulettes

Rattachement des auteurs :

1. Faculté de médecine, Université de Toronto, Toronto (Ontario), Canada

2. School of Population and Public Health, Université de la Colombie-Britannique, Vancouver (Colombie-Britannique), Canada

3. Département de pédiatrie, Université de la Colombie-Britannique, Vancouver (Colombie-Britannique), Canada

4. British Columbia Injury Research \& Prevention Unit, Vancouver (Colombie-Britannique), Canada

5. Child \& Family Research Institute, Vancouver (Colombie-Britannique), Canada

6. British Columbia Children's Hospital, Vancouver (Colombie-Britannique), Canada

Correspondance : Mariana Brussoni, F511-4480 Oak Street, Vancouver (Colombie-Britannique) V6H 3V4; tél. : 604-875-3712; téléc. : 604-875-3569; courriel : mbrussoni@cw.bc.ca 
et $49 \%$ des utilisateurs de trottinette consultaient pour des fractures ${ }^{3}$. De même, les données canadiennes ont fait ressortir des taux de fracture se situant entre 36,6\% et $48,1 \%$ chez les utilisateurs de dispositifs à petites roues 8 .

Des études antérieures ont utilisé les données sur les traumatismes de la surveillance des urgences, données tirées du Système canadien hospitalier d'information et de recherche en prévention des traumatismes (SCHIRPT), pour décrire les profils de blessure au Canada chez les cyclistes $^{7}$ et chez les utilisateurs de patins à roues alignées ${ }^{9}$. Les données relatives aux hospitalisations d'une étude canadienne réalisée en 2002 donnent à penser qu'il y a eu une diminution des blessures graves associées au cyclisme depuis l'implantation à grande échelle de lois sur le port du casque $^{10}$. Toutefois, à l'exception d'une étude de 2009 qui a utilisé les données du SCHIRPT de l'Alberta Children's Hospital pour examiner les blessures associées aux chaussures à roulettes et a comparé les profils de blessure et le port du casque pour d'autres activités sur roues, peu d'études ont comparé les profils de blessure ou le port du casque pour différentes activités sur roues ${ }^{6}$. Thakore et collab. ${ }^{6}$ ont fait état de blessures à la tête et au visage chez $15 \%$ à $22 \%$ des utilisateurs, sans spécification sur le type d'activité sur roues, et ils ont également constaté que les fractures étaient en réalité plus fréquentes chez les noncyclistes en Alberta.

Notre étude vise à analyser les données de surveillance des urgences du SCHIRPT afin de décrire l'épidémiologie des blessures associées aux activités non motorisées sur roues ainsi que le port du casque chez les patients pédiatriques se présentant aux urgences. Elle vise également à déterminer si le port du casque est fonction des dispositions législatives régionales. L'analyse des données recueillies aux urgences à l'échelle du Canada et la description des types de blessures subies par les utilisateurs de ces dispositifs peut aider à mieux cibler les stratégies de prévention des blessures. Les ensembles de données administratives du type des données de sommaires de départ ne prennent en compte qu'une fraction des cas de blessures, étant donné que la majorité des enfants blessés, en particulier ceux qui le sont le moins grièvement, ne sont pas hospitalisés. En outre, ces ensembles de données ne contiennent pas de description de l'accident ni ne font état de l'utilisation d'un équipement de protection, alors que les données de surveillance des urgences du SCHIRPT permettent ces niveaux d'analyse.

\section{Méthodologie}

Les données sur les patients ayant subi des blessures causées par des dispositifs à roues non motorisés ont été extraites du SCHIRPT pour les années 2004 à 2009. Le SCHIRPT est un programme national de surveillance des consultations aux urgences qui recense les cas de blessures traitées aux urgences de 11 hôpitaux pédiatriques et de 4 hôpitaux généraux au Canada, dans les villes de Vancouver (C.-B.), Calgary (Alb.), Edmonton (Alb.), Winnipeg (Man.), London (Ont.), Kingston (Ont., 2), Ottawa (Ont.), Montréal (Qc, 2), Québec (Qc), Toronto (Ont.), Halifax (N.-É.), St. John's (T.-N.-L.) et Arctic Bay (Nt) ${ }^{11}$. On demande à tout patient blessé qui se présente à l'hôpital, ou à la personne qui l'accompagne, de remplir un formulaire à propos de la blessure. Si le patient ou son représentant est incapable de remplir le formulaire, un membre du personnel le fait en se fondant sur des entrevues ou sur le dossier du patient. Les questions du formulaire portent sur les circonstances entourant l'accident, dont les facteurs qui ont causé la blessure ou qui y ont contribué ainsi que sur son moment et son lieu. Le diagnostic médical est codé par un coordonnateur local du SCHIRPT $^{11}$. Une fois remplis, les formulaires de déclaration des blessures sont envoyés au centre national du SCHIRPT, à l'Agence de la santé publique du Canada, où ils sont codés par un agent de soutien au traitement des données dûment formé.

Nos analyses portent sur les patients pédiatriques qui se sont présentés à un hôpital participant au SCHIRPT. Comme la majorité des hôpitaux participant au SCHIRPT sont des centres pédiatriques, et que nombre d'entre eux ne traitent que les patients âgés de moins de 17 ans, nous nous sommes intéressés uniquement aux patients âgés de 1 à 16 ans (12 à 203 mois). Un certain nombre de variables dans le SCHIRPT permettent de relever tous les cas liés à des activités non motorisées sur roues. Ces variables sont l' " activité au moment de la blessure » (contexte), les " codes de facteur» et les " champs descriptifs " (description de l'accident et conséquences). Les cas ont été retenus si l'activité au moment de la blessure était codée comme étant la pratique de la bicyclette (incluant le monocycle et le tricycle), de la planche à roulettes, du patin à roues alignées / à roulettes ou de la trottinette, si une valeur associée à l'un ou l'autre des 4 dispositifs à roues non motorisés figurait dans l'un des 6 champs utilisés pour indiquer le code de facteur ou si les champs descriptifs contenaient l'une des chaînes de caractères (en français ou en anglais) associées à ces 4 activités/dispositifs. Différents types ont été inclus pour chaque dispositif. Les monocycles et les tricycles ont été comptabilisés comme des bicyclettes et aucune distinction n'a été faite entre les patins à roulettes et les patins à roues alignées. Après réception des données codées du centre national du SCHIRPT, chaque chaîne de caractères descriptive de l'ensemble de données a été examinée. Si le texte descriptif indiquait que la blessure ne s'était pas produite pendant l'utilisation d'un dispositif à roues ou que le dispositif en question était soit un dispositif motorisé soit une poussette, l'entrée a été exclue. On a considéré que le blessé portait un casque au moment de l'accident lorsque le patient ou la personne responsable avait coché la case " casque » à la question du formulaire du SCHIRPT portant sur l'utilisation d'un équipement de protection. On a considéré qu'un équipement de protection avait été utilisé si l'une au moins des autres options avaient été cochée à la même question (" rembourrage de protection ", " protègedents », etc.). La méthodologie de l'étude a été approuvée par le comité d'éthique de la recherche du Children's and Women's Hospital de la Colombie-Britannique.

Sur les huit provinces représentées dans le SCHIRPT, cinq possédaient des dispositions législatives (une loi provinciale ou un règlement municipal applicable au lieu où est situé l'hôpital participant au SCHIRPT, par exemple St. John's, à Terre-Neuve-et-Labrador) rendant obligatoire le port du casque au cours des années sur lesquelles portait l'analyse. 
Toutes les dispositions législatives portant sur le port du casque à bicyclette avaient été adoptées avant 2004, et aucune autre région n'a adopté de nouvelles lois au cours de la période, même si, en 2007, la Nouvelle-Écosse a étendu la portée de sa loi sur le port du casque à toutes les activités sur roues ${ }^{12}$. Dans deux provinces, la Colombie-Britannique et la Nouvelle-Écosse, le port du casque était obligatoire pour tous, indépendamment de l'âge, alors que cette obligation s'appliquait uniquement aux personnes de moins de 18 ans en Alberta et en Ontario, et aux enfants de moins de 12 ans à St. John's.

On a comparé les quatre types d'activités sur roues en fonction de l'âge médian et du sexe. Les fréquences des profils de blessure (selon la définition figurant dans l'ensemble de données du SCHIRPT et l'indication par le médecin traitant), dont les "fractures », les "luxations » et les " traumatismes crâniens ", ont été comparées par type d'activité. La fréquence de port du casque ou d'un équipement de protection a aussi été comparée selon le type d'activité. La gestion et les analyses des données ont été effectuées à l'aide du logiciel Microsoft Excel (Microsoft, 2010) et de la version 20.0 du logiciel SPSS (IBM, 2011). On a eu recours aux rapports de cote pour faire ressortir les différences significatives sur le plan des fréquences. Les rapports de cotes ont été calculés par régression logistique binaire, avec ajustement pour tenir compte de l'âge, du sexe et de l'activité non motorisée sur roues, et avec des intervalles de confiance (IC) à $95 \%$. On a utilisé une régression de Poisson généralisée pour les tendances temporelles, afin de quantifier la relation entre l'année et le nombre de blessures survenues. Afin de tenir compte de la surdispersion des données chiffrées, nous avons utilisé un chi carré de Pearson avec un IC à $95 \%$ pour estimer le paramètre d'échelle et obtenir des estimations de la variance et des seuils de signification plus prudents ${ }^{13}$. Les estimations exponentielles $\beta$, qui indiquent le changement relatif du nombre de blessures par année, sont calculées à partir de ce modèle de régression. La régression tient pour acquis que le dénominateur (nombre de personnes à risque de blessures) n'a pas changé avec le temps.

\section{Résultats}

\section{Données relatives à l'âge et au sexe}

Entre 2004 et 2009, il y a eu au total 28618 enregistrements relatifs à des patients blessés qui satisfaisaient aux critères d'inclu- sion, ce qui représente 28618 patients ayant subi 35184 blessures au cours d'activités non motorisées sur roues. Parmi ces patients, 72,8 \% (20 838/28 618) avaient subi des blessures liées à la pratique de la bicyclette, $17,1 \%(4892 / 28$ 618) à celle de la planche à roulettes, 6,7 \% (1904/28 618) à celle de la trottinette et $3,1 \%(891 / 28$ 618) à celle du patin à roues alignées.

Le tableau 1 présente la répartition des blessures selon le sexe et l'âge pour les diverses activités non motorisées sur roues. La majorité des blessures ont été subies par des garçons (74,1\%). Ils ont été blessés dans une plus forte proportion que les filles pour toutes les activités, que le rapport entre le nombre de garçons et le nombre de filles soit élevé $(8,2$ pour la planche à roulettes) ou faible $(1,4$ pour la trotinette). La plupart des enfants présentant des blessures associées à des activités non motorisées sur roues étaient âgés de 9 à 12 ans $(37,2 \%)$, mais la répartition selon l'âge variait de manière significative selon le sport : les enfants présentant des blessures liées à l'utilisation de la trottinette étaient plus jeunes (âge médian de 9,4 ans), les cyclistes étaient un peu plus âgés (10,9 ans), puis venaient les enfants circulant en patins à roues alignées $(11,7$ ans) et enfin les utilisateurs de

TABLEAU 1

Répartition des patients ( 1 à 16 ans) selon le sexe et l'âge et en fonction du type d'activité non motorisée sur roues, 2004 à 2009

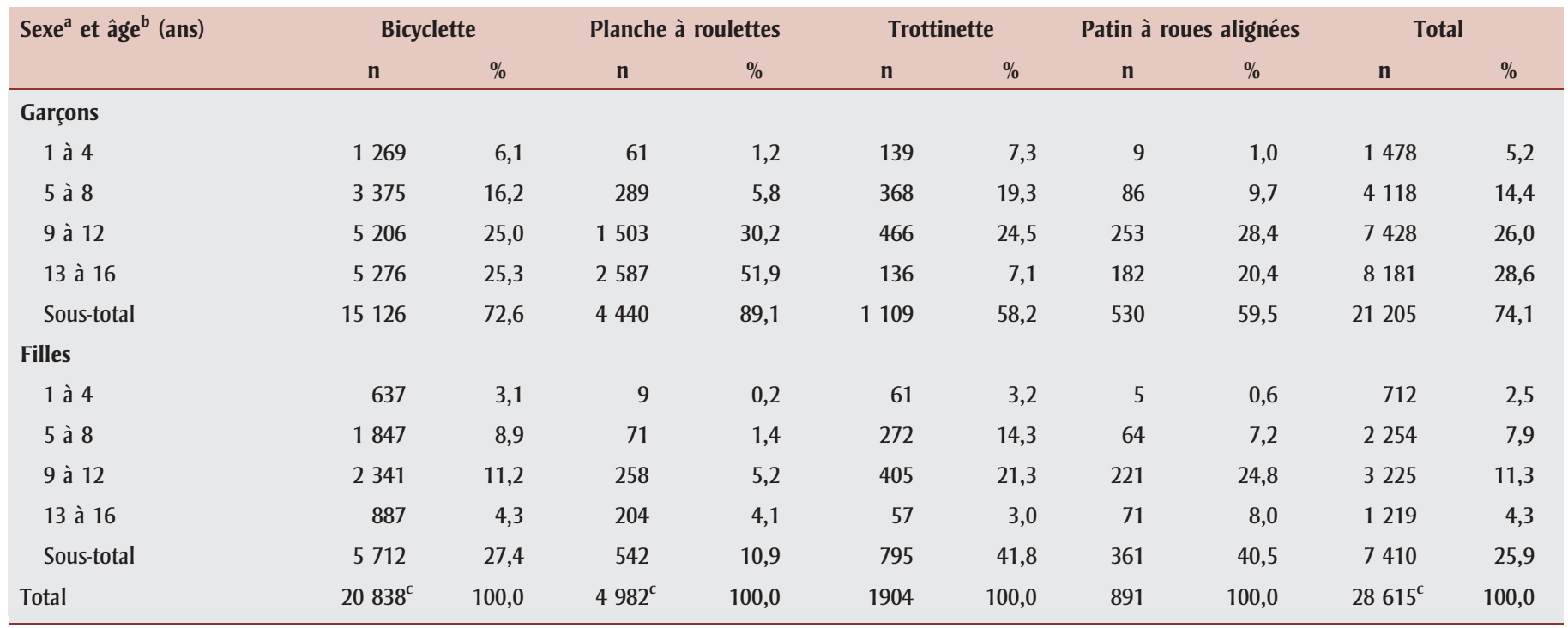

Abréviation : dl, degrés de liberté.

a Sexe selon l'activité : $\chi^{2}=959,2, d l=3, p<0,001$.

b Âge selon l'activité : $\chi^{2}=2375,4, d l=9, p<0,001$.

c Les données sur le sexe étaient manquantes pour 3 patients ( 1 cycliste et 2 utilisateurs de planche à roulettes). 
planche à roulettes de (13,3 ans, groupe généralement le plus âgé).

Tendances temporelles des blessures subies roues

Pour toutes les activités non motorisées sur roues, les blessures atteignaient un sommet au cours des mois d'été : entre $40 \%$ et $50 \%$ des blessures étaient survenues entre juillet et septembre pour les 6 années. En général, le nombre de consultations pour blessures causées par des activités non motorisées sur roues a diminué de manière significative entre 2004 et 2009 (figure 1), en moyenne de $6,0 \%$ annuellement $(\exp (\mathrm{B})=0,94$; IC à $95 \%: 0,92$ à 0,96$)$. Tous les types de blessures subies au cours d'activités non motorisées sur roues ont diminué de manière significative au fil des ans, à l'exception des blessures chez les utilisateurs de trottinette $(\exp (B)=1,00$; IC à $95 \%: 0,97$ à 1,03) : chaque année, le nombre de blessés a diminué en moyenne d'environ 5,1 \% chez les cyclistes $(\exp (\mathrm{B})=0,95$; IC à $95 \%$ : 0,93 à 0,97 ), de $9,0 \%$ chez les utilisateurs de planche à roulettes $(\exp (\mathrm{B})=0,91$; IC à $95 \%$ : 0,86 à 0,96) et d'environ 21,5\% chez les utilisateurs de patins à roues alignées $(\exp (B)=0,79$; IC à $95 \%: 0,69$ à 0,90). au cours d'activités non motorisées sur

\section{Profils de blessure associés à des activités non motorisées sur roues}

Les mécanismes lésionnels associés aux activités non motorisées sur roues étaient similaires pour toutes les activités, les chutes étant le mécanisme le plus fréquent (82\% à $91 \%$; données non présentées). Les collisions et les collisions avec un véhicule motorisé étaient les deux autres principaux mécanismes lésionnels. Ces dernières touchaient 5,9\% des cas chez les cyclistes et variaient entre 1,7 \% et 4,9\% pour les autres activités.

En général, les types de blessure les plus fréquents étaient les blessures superficielles et les fractures, suivies des blessures musculosquelettiques, comme les entorses (tableau 2). C'est chez les utilisateurs de patins à roues alignées qu'on observait la plus forte proportion de fractures $(46,1 \%)$. Les blessures graves, en particulier celles touchant un organe interne, les lésions neurovasculaires ou les blessures multiples, étaient plus nombreuses chez les cyclistes. Les traumatismes crâniens représentaient plus de 8,3\% de l'ensemble des blessures, et touchaient davantage les cyclistes. On a observé la plus forte proportion de blessures musculosquelettiques, comme les entorses, chez les utilisateurs de planche à roulettes, et la

FIGURE 1

Nombre annuel de consultations pour blessures associées à des activités non motorisées sur roues, patients de 1 à 16 ans, 2004 à 2009

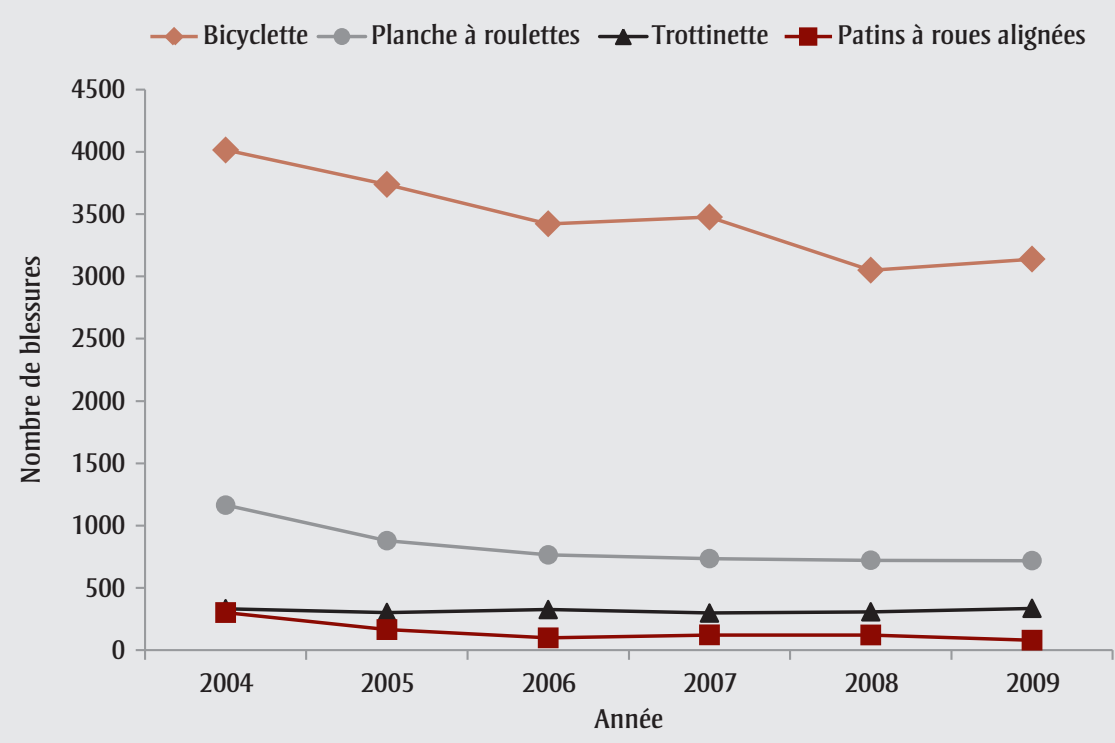

plus forte proportion de blessures aux dents chez les enfants utilisant une trottinette.

Pour toutes les activités, le type de fracture le plus fréquent était la fracture du coude ou de l'avant-bras, suivie de la fracture du poignet ou de la main (tableau 3). Les utilisateurs de planche à roulettes sont ceux qui avaient subi le plus grand nombre et la plus forte proportion de fractures de la cheville ou du pied.

\section{Port du casque et d'un équipement de protection}

Parmi les enfants présentant une blessure subie au cours d'une activité non motorisée sur roues, 55,3\% ont déclaré qu'ils portaient un casque (données manquantes exclues; données non présentées). Dans les cas où les données sur le port du casque étaient manquantes, les proportions des enregistrements selon le sexe, la présence d'un traumatisme crânien et l'hospitalisation étaient semblables (à $3 \%$ près) à celles observées lorsque le port du casque était déclaré. Les enfants de 1 à 4 ans représentaient une proportion supérieure $(12,3 \%$ contre $6,0 \%$ ) des enregistrements pour lesquels les données sur le port du casque étaient manquantes et les cyclistes une moindre proportion (68,8 \% contre $74,3 \%)$ de ces enregistrements.

Le port du casque variait en fonction de l'activité non motorisée sur roues. C'est chez les cyclistes $(62,2 \%)$ qu'il était le plus répandu, puis chez les utilisateurs de patins à roues alignées $(47,1 \%)$, de trottinette $(39,7 \%)$ et de planche à roulettes $(32,9 \%)$. Seulement $4,1 \%$ de l'ensemble des patients avaient utilisé un autre équipement de protection, et cela concernait surtout les utilisateurs de patins à roues alignées $(12,7 \%)$. Pour toutes les activités et sans distinction d'âge ou de sexe, les enfants qui portaient un casque étaient moins nombreux à avoir subi un traumatisme crânien que ceux qui n'en portaient pas (tableau 4).

Parmi l'ensemble des patients ayant présenté des blessures subies au cours d'activités non motorisées sur roues, 2314 $(8,1 \%)$ avaient été hospitalisés. Parmi 
TABLEAU 2

Type de blessure en fonction de l'activité non motorisée sur roues, patients de 1 à 16 ans, 2004 à 2009

\begin{tabular}{|c|c|c|c|c|c|c|}
\hline Type de blessure & $\begin{array}{c}\text { Bicyclette } \\
\text { n (\%) }\end{array}$ & $\begin{array}{l}\text { Planche à roulettes } \\
\text { n (\%) }\end{array}$ & $\begin{array}{l}\text { Trottinette } \\
\text { n (\%) }\end{array}$ & $\begin{array}{c}\text { Patin à roues alignées } \\
\text { n (\%) }\end{array}$ & $\begin{array}{l}\text { Total }^{\mathrm{a}} \\
\text { n (\%) }\end{array}$ & $\chi^{2}(d I, p)^{b}$ \\
\hline Blessures superficielles & $10036(38,0)$ & $974(17,4)$ & $734(33,2)$ & $211(21,8)$ & $11955(34,0)$ & \\
\hline Fractures & $7446(28,2)$ & $2315(41,5)$ & $766(34,6)$ & $447(46,1)$ & $10974(31,2)$ & $514,2(3,<0,001)$ \\
\hline Blessures musculosquelettiques ${ }^{c}$ & $4836(18,3)$ & $1765(31,6)$ & $449(20,3)$ & $231(23,8)$ & $7281(20,7)$ & \\
\hline Traumatisme crânien (mineur) ${ }^{\mathrm{d}}$ & $2278(8,6)$ & $293(5,2)$ & $122(5,5)$ & $40(4,1)$ & $2733(7,8)$ & $110,1(3,<0,001)$ \\
\hline Traumatisme crânien (grave) ${ }^{\mathrm{e}}$ & $166(0,6)$ & $27(0,5)$ & $7(0,3)$ & $3(0,3)$ & $203(0,6)$ & $5,8(3,<0,12)$ \\
\hline Blessures aux dents & $701(2,7)$ & $49(0,9)$ & $77(3,5)$ & $4(0,4)$ & $831(2,4)$ & \\
\hline Blessures multiples ou graves ${ }^{f}$ & $420(1,6)$ & $35(0,6)$ & $10(0,5)$ & $4(0,4)$ & $469(1,3)$ & $53,6(3,<0,001)$ \\
\hline Luxation & $143(0,5)$ & $52(0,9)$ & $9(0,4)$ & $9(0,9)$ & $213(0,6)$ & \\
\hline Autres $^{g}$ & $395(1,5)$ & $73(1,3)$ & $37(1,7)$ & $20(2,1)$ & $525(1,5)$ & \\
\hline Total $^{\mathrm{a}}$ & $26421(100)$ & $5583(100)$ & $2211(100)$ & 969 (100) & $35184(100)$ & \\
\hline
\end{tabular}

Abréviation : dl, degrés de liberté.

${ }^{a}$ Les chiffres représentent le nombre total de blessures; les patients peuvent présenter plus d'une blessure.

b $\chi^{2}$ compare les proportions entre les divers types d'activité.

c Blessures musculosquelettiques, p. ex. entorses.

d Traumatismes crâniens mineurs comprennent les commotions cérébrales et les blessures à la tête mineures.

e Traumatismes crâniens graves comprennent les traumatismes intracrâniens.

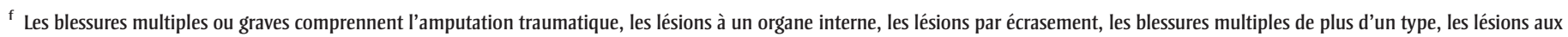
vaisseaux sanguins et aux nerfs.

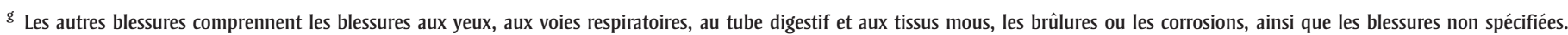

les patients pour lesquels les données sur le port du casque étaient disponibles, ceux qui avaient été hospitalisés avaient été significativement moins nombreux à porter un casque, sans distinction d'âge ou de sexe, comparativement à ceux qui avaient été autorisés à rentrer à la maison. Lorsque ces données ont été analysées en fonction du type d'activité, toutefois, cette différence n'était significative que chez les cyclistes (tableau 4).

\section{Port du casque et réglementation à cet égard}

Chez les patients qui s'étaient présentés aux urgences en raison d'une blessure associée à un dispositif à roues non motorisé dans les régions où le port du casque était obligatoire, la probabilité d'avoir subi un traumatisme crânien était significativement plus faible $(\mathrm{RC}=0,86$; IC à $95 \%: 0,80$ à 0,94$)$, sans distinction d'âge ou de sexe (tableau 5). Il existait également une association entre la réglementation et le port du casque, dans la mesure où les patients ayant consulté dans

TABLEAU 3

Type de fracture selon l'activité non motorisée sur roues, patients de 1 à 16 ans, 2004 à 2009

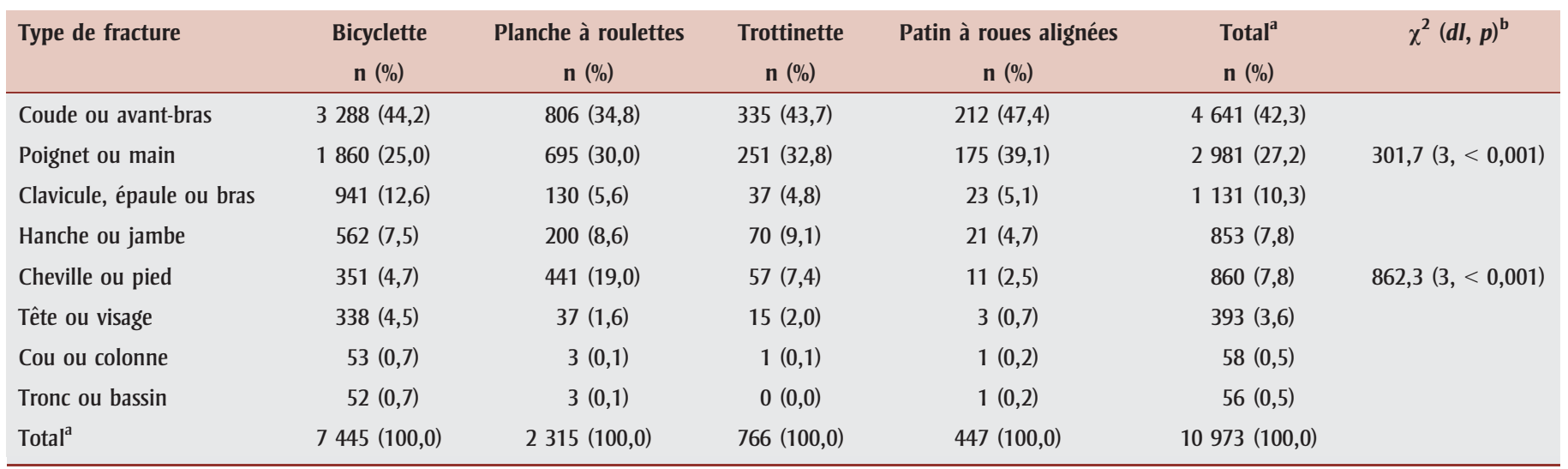

Abréviation : dl, degrés de liberté.

Remarque : Les données sur le type de blessure étaient manquantes pour 1 cycliste.

a Les chiffres représentent le nombre de blessures; les patients peuvent présenter plus d'une blessure.

b $\chi^{2}$ compare les proportions pour les divers types d'activité. 
TABLEAU 4

Type de blessure selon l'activité non motorisée sur roues et le port du casque, patients de 1 à 16 ans, 2004 à 2009

\begin{tabular}{|c|c|c|c|c|c|c|}
\hline \multirow[t]{2}{*}{ Activité } & \multicolumn{3}{|c|}{ Traumatisme crânien } & \multicolumn{3}{|c|}{ Hospitalisation } \\
\hline & $\begin{array}{c}\text { Port du casque }^{\mathrm{a}} \\
\text { n (\%) }\end{array}$ & $\begin{array}{c}\text { Pas de casque } \\
\text { n (\%) }\end{array}$ & $\begin{array}{l}\text { RC ajusté } \\
\text { (IC à } 95 \%)\end{array}$ & $\begin{array}{c}\text { Port du casque } \\
\text { n (\%) }\end{array}$ & $\begin{array}{c}\text { Pas de casque } \\
\text { n (\%) }\end{array}$ & $\begin{array}{l}\text { RC ajustéb } \\
\text { (IC à } 95 \%)\end{array}$ \\
\hline Planche à roulettes & $39(16,5)$ & $198(83,5)$ & $0,33(0,23$ à 0,46$)$ & $56(29,9)$ & $131(70,1)$ & $0,78(0,56$ à 1,07$)$ \\
\hline Trottinette & $24(27,6)$ & $63(72,4)$ & $0,53(0,33$ à 0,86$)$ & $18(40,0)$ & $27(60,0)$ & $0,99(0,55$ à 1,85$)$ \\
\hline
\end{tabular}

Abréviations : dl, degrés de liberté; IC, intervalle de confiance; RC, rapport de cote.

a Port du casque selon l'activité : $\chi^{2}=1185,3, d l=3, p<0,001$.

${ }^{\mathrm{b}} \mathrm{RC}$ ajusté pour l'âge et le sexe.

une région avec ce type de réglementation étaient 2,12 fois plus nombreux à déclarer porter un casque (IC à $95 \%: 1,99$ à 2,26).

\section{Analyse}

À notre connaissance, notre étude est la première étude épidémiologique dans laquelle on compare les blessures chez les utilisateurs de dispositifs à roues non motorisés à l'échelle du Canada. Elle révèle que ces blessures sont à l'origine d'une proportion appréciable de la morbidité et des consultations aux urgences chez les patients pédiatriques et elle fournit des preuves convaincantes de l'importance de la réglementation sur le port du casque pour la prévention des blessures.

Le cyclisme était à l'origine de la plupart des blessures, probablement en raison de sa popularité : une enquête canadienne a révélé que $94 \%$ des enfants de 5 à 12 ans et $79 \%$ des enfants de 13 à 17 ans avaient fait de la bicyclette au cours des 12 mois précédents $^{14}$. Selon un sondage annuel effectué aux États-Unis, 39,8 \% des participants âgés de plus de 7 ans ont déclaré avoir fait de la bicyclette, 7,7 \%, de la planche à roulettes, 7,5 \% du patin à roues alignées et $7,4 \%$, de la trottinette ${ }^{15}$. D’après les mêmes données, les garçons représentaient $76 \%$ des utilisateurs de planche à roulettes, $51 \%$ des utilisateurs de patins à roues alignées, $56 \%$ des cyclistes et $58 \%$ des utilisateurs de trottinette ${ }^{16}$. Toutefois, ils constituaient une majorité disproportionnée des blessés pour l'ensemble des activités non motorisées sur roues. Cette prédominance pourrait être attribuable au fait que les garçons participent davantage à certaines activités ${ }^{17}$ ou à des différences sur le plan des comportements à risque ${ }^{18}$. Même si l'on dispose de peu de données sur l'âge des utilisateurs de dispositifs à roues, la majorité des études antérieures sur les profils de blessure ont fait ressortir un profil selon l'âge similaire : les patients ayant subi des blessures associées à l'utilisation d'une trottinette étaient en général plus jeunes, ceux présentant des blessures associées à la planche à roulettes généralement plus âgés ${ }^{3,6}$. Toutefois, les études incluant des données sur les adultes laissent penser que l'utilisation de la planche à roulettes et de la trottinette est plus fréquente chez les enfants ${ }^{1,15}$. Presque toutes les études montrent que la majorité des consultations pour blessures subies au cours d'activités non motorisées sur roues concernent des enfants de 9 à 14 ans $^{3,6,19}$. La diminution observée dans

TABLEAU 5

Port du casque et traumatisme crânien associé aux activités non motorisées sur roues, selon la réglementation sur le port du casque, patients de 1 à 16 ans, 2004 à 2009

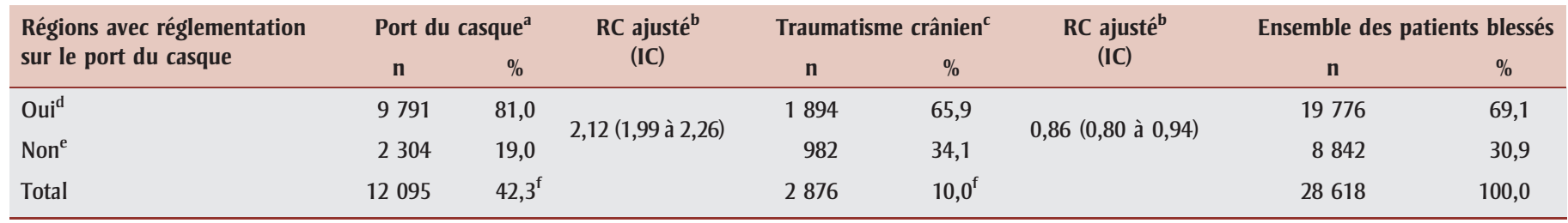

Abréviations : dl, degrés de liberté; IC, intervalle de confiance; RC, rapport de cote.

${ }^{a}$ Port du casque en fonction de la réglementation : $\chi^{2}=491,6, d l=1, p<0,001$; les données sur le port du casque excluent les patients pour lesquels aucune donnée à ce sujet n'est disponible.

b RC ajusté pour l'âge, le sexe et l'activité non motorisée sur roues.

c Traumatisme crânien par province: $\chi^{2}=15,6, d l=1, p<0,001$.

${ }^{\text {d }}$ Régions avec réglementation : Colombie-Britannique, Alberta, Ontario, Nouvelle-Écosse et St. John's (Terre-Neuve-et-Labrador).

e Régions sans réglementation : Nunavut, Manitoba, Québec.

f Pourcentage par rapport à l'ensemble des patients blessés. 
le nombre de consultations pour blessures chez les cyclistes pourrait être due à une diminution de l'utilisation au cours de la période $^{20}$ ou à l'efficacité des mesures de prévention des blessures.

Les chutes étaient beaucoup plus fréquentes que les autres mécanismes lésionnels, toutes activités confondues. Les collisions avec un véhicule motorisé étaient à l'origine d'une très faible proportion des blessures, même celles associées au cyclisme. Toutefois, comme les cas de blessures graves ne passent généralement pas par les urgences, il est fort possible qu'ils soient mal représentés dans le SCHIRPT. Quoi qu'il en soit, nos résultats laissent penser que, même si les compétences en matière de circulation routière sont importantes pour assurer la sécurité des enfants, le port de casque et d'autres équipements protecteurs visant à protéger les enfants en cas de chute est justifié, tout comme les améliorations techniques réduisant la probabilité des chutes. Des données probantes indiquent que les protège-poignets, les protège-coudes et les genouillères peuvent prévenir certaines blessures, en particulier les fractures du poignet et de l'avant-bras, les blessures aux coudes et les blessures aux genoux $^{21,22}$. Dans notre étude, les blessures les plus fréquentes, toutes activités confondues, étaient les fractures, en particulier les fractures du coude ou de l'avantbras, suivies des fractures du poignet ou de la main, observation qui concorde avec celles d'autres études ${ }^{6}$. Il y a donc tout lieu de croire que bon nombre des blessures subies au cours d'activités sur roues pourraient être prévenues par le port d'équipement de protection, mesure qui est d'ailleurs recommandée par l'American Association of Pediatrics ${ }^{23}$. Toutefois, le port d'équipement de protection en général était peu fréquent pour l'ensemble des activités, ce qui plaide en faveur d'une intervention auprès de cette population à risque.

Les traumatismes crâniens demeurent une cause importante de morbidité associée aux activités non motorisées sur roues. On en observe la plus forte proportion chez les cyclistes, la plus faible chez les utilisateurs de patins à roues alignées. Heureusement, le taux de traumatismes crâniens graves demeure faible, quoique la proportion des traumatismes crâniens graves soit la même quelle que soit l'activité, ce qui porte à croire que le risque de subir un traumatisme crânien grave demeure important indépendemment du type d'activité non motorisée sur roues. En outre, grâce à une meilleure compréhension des commotions cérébrales et des traumatismes crâniens associés au sport ${ }^{24,25}$, les consultations pour blessures à la tête, même mineures, offrent une occasion de réduire les conséquences à long terme de ces blessures grâce à une éducation appropriée.

Nos résultats laissent penser que le port du casque est associé à une diminution de la probabilité aussi bien des traumatismes crâniens que des hospitalisations. De plus, le fait que les taux de port du casque soient nettement plus élevés et que la probabilité de subir un traumatisme crânien soit plus faible dans les hôpitaux des régions où le port du casque est obligatoire laisse croire que la réglementation à ce sujet en est la cause. Une étude canadienne antérieure a montré que la réglementation sur le port du casque diminue la fréquence des traumatismes crâniens chez les cyclistes et que la pratique du cyclisme augmente chez les enfants après l'adoption d'une telle réglementation. C'est donc dire que l'effet protecteur du casque n'est pas attribuable à une baisse de la pratique du cyclisme ${ }^{10}$. En 2010, six provinces disposaient d'une loi rendant obligatoire le port du casque chez les cyclistes de moins de 18 ans, même si la mise en application de cette loi était variable d'une région à l'autre et aurait été difficile à mesurer ${ }^{12}$. Il n'y a pas de loi à cet égard dans les sept autres provinces/ territoires, même si certaines villes peuvent avoir des règlements rendant obligatoire le port du casque, comme St. John's (T.-N.-L.), ville où il y a un hôpital participant au SCHIRPT (et même si certains patients peuvent venir de l'extérieur de la région visée par ce règlement). La Nouvelle-Écosse est la seule province à avoir adopté une loi rendant obligatoire le port du casque pour toutes les activités non motorisées sur roues ${ }^{12}$.

Le port du casque dans notre étude n'était globalement que de 55 \% (au sein des cas pour lesquels les données étaient disponibles), résultat qui concorde avec les taux calculés de manière similaire dans d'autres études ${ }^{6}$, dont les données déclarées par le SCHIRPT ${ }^{8}$. Ce constat pourrait être attribuable au fait que les enfants portant un casque risquent moins d'être blessés et, par conséquent, de se présenter aux urgences. Il pourrait également être lié à la méthode de collecte de données dans chaque hôpital.

\section{Points forts et limites}

Comme nous avons utilisé des données rétrospectives, nos données et nos inférences sont limitées par la manière dont les formulaires ont été remplis par les patients et les médecins. La principale limite de cette étude tient au fait que nous ne pouvions pas calculer les taux de blessures pour l'ensemble des utilisateurs de dispositifs à roues non motorisés. Le nombre de blessures observées dépend dans une large mesure de la fréquence d'utilisation de chaque dispositif et n'est pas représentatif de l'incidence des blessures pour l'activité. En outre, les patients qui se présentent aux hôpitaux participant au SCHIRPT pourraient ne pas être représentatifs de l'ensemble des utilisateurs de dispositifs à roues non motorisés. La majorité des hôpitaux participant au SCHIRPT sont situés en milieu urbain et ne reçoivent qu'un petit nombre de l'ensemble des blessés qui consultent pour obtenir des soins : les patients qui se présentent dans d'autres hôpitaux, dans des cliniques sans rendezvous ou au cabinet de leur médecin de famille ne sont pas pris en compte. Toutefois, la recherche laisse penser que les données du SCHIRPT qui concernent les sports et les activités récréatives en particulier peuvent être représentatives de la population canadienne $e^{26,27}$. En outre, l'utilisation de données nationales sur une période de six ans augmente la puissance statistique de nos données, et les données de surveillance du SCHIRPT constituent la plus importante source de données disponible sur les urgences à l'échelle du Canada, ce qui les rend utiles pour décrire l'épidémiologie des blessures. 


\section{Conclusion}

Notre étude, comme d'autres recherches, vont dans le sens des dispositions législatives rendant obligatoire le port du casque, car ces dernières constituent un moyen efficace de réduire les blessures chez les utilisateurs de dispositifs à roues non motorisés. De plus, les consultations aux urgences peuvent offrir une occasion unique de prévenir les blessures : des travaux antérieurs ont révélé que les patients étaient à cette occasion réceptifs à l'information sur la prévention des blessures $^{28}$ et plus susceptibles de modifier leurs comportements après avoir reçu des conseils aux urgences ${ }^{29}$. Des études ont montré que l'utilisation des dispositifs de sécurité augmente lorsqu'on fournit des casques ou des équipements de protection (comme des sièges d'appoint) ou que l'on facilite l'accès à ces produits ${ }^{30,31}$. Les cas de blessures chez les enfants qui se présentent aux urgences pourraient être une occasion unique de parler des mesures de sécurité et d'encourager l'observance des recommandations à cet égard.

\section{Remerciements}

Mariana Brussoni détient une bourse de chercheur émérite (soutien à la carrière) de la Michael Smith Foundation for Health Research (MSFHR) et une bourse salariale du British Columbia Child and Family Research Institute. Les données figurant dans la présente publication ont été utilisées avec l'autorisation de l'Agence de la santé publique du Canada. Nous souhaitons remercier de son aide la section des blessures de la Division de la surveillance de la santé et de l'épidémiologie, qui a facilité l'obtention des données. Les analyses et les interprétations présentées dans cet article ne reflètent pas nécessairement les opinions du gouvernement fédéral. Nous souhaitons également remercier la $\mathrm{D}^{\mathrm{re}}$ Aybaniz Ibrahimova pour sa contribution à l'analyse des données.

\section{Références}

1. Konkin DE, Garraway N, Hameed $\mathrm{S}$ et collab. Population-based analysis of severe injuries from motorized wheeled vehicles. Am J Surg. 2006;191:615-8.
2. Institut canadien d'information sur la santé (ICIS). Nombre d'hospitalisations au Canada en raison d'une blessure liée au cyclisme, 2009-2010. Ottawa : ICIS; 2011.

3. Brudvik C. Injuries caused by small wheel devices. Prev Sci. 2006;7:313-20.

4. Banas MP, Dalldorf PG, Marquardt JD. Skateboard and in-line skate fractures: a report of one summer's experience. J Orthop Trauma. 1992;6:301-5.

5. Zalavras C, Nikolopoulou G, Essin D, Manjra N, Zionts LE. Pediatric fractures during skateboarding, roller skating, and scooter riding. Am J Sports Med. 2005;33:568-73.

6. Thakore S, Tram J, Hagel BE, Kyle T, Senger T, et F Belanger. Les blessures chez les utilisateurs de chaussures à roulettes : Une comparaison avec les autres activités non motorisées sur roues. Pediatr Child Health. 2009;14,8:509-513.

7. Linn S, Smith D, Sheps S. Epidemiology of bicycle injury, head injury, and helmet use among children in British Columbia: a five year descriptive study. Inj Prev. 1998;4: $122-5$.

8. Section des blessures et de la violence à l'égard des enfants. Surveillance des services d'urgence - Blessures associées aux dispositifs à roues non motorisés. Le Système canadien hospitalier d'information et de recherche en prévention des traumatismes (SCHIRPT), tous les âges, 19902007. Ottawa (Ont.) : Agence de la santé publique du Canada; 2011.

9. Ellis JA, Kierulf JC, Klassen TP. Injuries associated with in-line skating from the Canadian Hospitals Injury Reporting and Prevention Program database. Can J Public Health. 1995;86:133-6.

10. Macpherson AK, To TM, Macarthur C, Chipman ML, Wright JG, Parkin PC. Impact of mandatory helmet legislation on bicyclerelated head injuries in children: a population-based study. Pediatrics 2002;110: e60-e60.

11. Mackenzie SG, Pless IB. CHIRPP: Canada's principal injury surveillance program. Inj Prev. 1999;5:208-13.
12. ThinkFirst - Pensez d'abord. Snapshot of bicycle helmet legislation [Internet]. ThinkFirst; 2010 [consultation le 16 avril 2013]. PDF (32,26 Ko) téléchargeable à partir du lien : http://www.thinkfirst.ca/documents /Snapshotofhelmetlegislation.pdf

13. McCullagh P, Nelder JA. Generalized linear models. $2^{\mathrm{e}}$ éd. London (UK) : Chapman \& Hall; 1989.

14. Craig CL, Cameron C, Russell SJ, et A Beaulieu. Créer un système propice des sports et des loisirs pour accroître l'activité physique [Internet]. Ottawa, Institut canadien de la recherche sur la condition physique et le mode de vie, 2001 [consultation le 8 juillet 2013]. PDF (748,39 Ko) téléchargeable à partir du lien : http:// www.cflri.ca/media/node/427/files/siap99 .pdf

15. National Sporting Goods Association. 2010 sports participation: ranked by total participation [Internet]. Mount Prospect (IL) : NSGA; 2010 [consultation le 16 avril 2013]. PDF (38,33 Ko) téléchargeable à partir du lien : http://www.nsga.org/files/public/2010 Participation_Ranked_by_TotalParticipation _4Web.pdf

16. National Sporting Goods Association. 2010 women's participation: Ranked by total female participation [Internet]. Mount Prospect (IL) : NSGA; 2010 [Consultation le 16 avril 2013]; PDF téléchargeable à partir du lien : http://www.nsga.org/files/public /2010Women'sParticipation-RankedbyTotal FemaleParticipation_4Web.pdf

17. Leslie E, Kremer P, Toumbourou JW, Williams JW. Gender differences in personal, social and environmental influences on active travel to and from school for Australian adolescents. J Sci Med Sport. 2010;13:597-601.

18. Morrongiello BA, Rennie H. Why do boys engage in more risk taking than girls? The role of attributions, beliefs, and risk appraisals. J Pediatr Psychol. 1998;23:33-43.

19. Hassan I, Dorani BJ. Rollerblading and skateboarding injuries in children in northeast England. J Accid Emerg Med. 1999; 16:348-50. 
20. National Sporting Goods Association. Tenyear history of sports participation [Internet]. Mount Prospect (IL) : NSGA; 2010. PDF (56,21 Ko) téléchargeable à partir du lien : http://www.nsga.org/files/public/Ten-Year _History_of_Sports_Participation_4web_100521 .pdf

21. Russell K, Hagel B, Francescutti LH. The effect of wrist guards on wrist and arm injuries among snowboarders: a systematic review. Clin J Sport Med. 2007;17:145-50.

22. Schieber RA, Branche-Dorsey CM, Ryan GW, Rutherford Jr. GW, Stevens JA, O’Neil J. Risk factors for injuries from in-line skating and the effectiveness of safety gear. N Engl J Med. 1996;335:1630-5.

23. Committee on Injury and Poison Prevention; American Academy of Pediatrics. Skateboard and scooter injuries. Pediatrics 2002;109: 542-3.

24. Broglio SP, Puetz TW. The effect of sport concussion on neurocognitive function, self-report symptoms and postural control: a meta-analysis. Sports Med. 2008;38:5367.

25. Yeates KO. Mild traumatic brain injury and postconcussive symptoms in children and adolescents. J Int Neuropsychol Soc. 2010;16:953-60.

26. Kang J, Hagel B, Emery CA, Senger T, Meeuwisse W. Assessing the representativeness of Canadian Hospitals Injury Reporting and Prevention Programme (CHIRPP) sport and recreational injury data in Calgary, Canada. Int J Inj Contr Saf Promot. 2013;20:19-26.

27. Pickett W, Brison RJ, Mackenzie SG, et al. Youth injury data in the Canadian Hospitals Injury Reporting and Prevention Program: do they represent the Canadian experience? Inj Prev. 2000;6:9-15.

28. Gittelman MA, Pomerantz WJ, Fitzgerald MR, Williams K. Injury prevention in the emergency department: a caregiver's perspective. Pediatr Emerg Care. 2008;24:5248.

29. Fernandez WG, Winter MR, Mitchell PM et collab. Six-month follow-up of a brief intervention on self-reported safety belt use among emergency department patients. Acad Emerg Med. 2009;16:1221-4.
30. Bishai D. Contracting with children and helmet distribution in the emergency department to improve bicycle helmet use. Acad Emerg Med. 2003;10:1371-7.

31. Gittelman MA, Pomerantz WJ, Frey LK. Use of a safety resource center in a pediatric emergency department. Pediatr Emerg Care. 2009;25:429-33. 